\title{
A Distributed Partial Order Reduction Algorithm
}

\author{
Robert Palmer and Ganesh Gopalakrishnan ${ }^{\star}$ \\ University of Utah, School of Computing \\ \{rpalmer, ganesh\}@cs.utah.edu
}

A distributed version of the PV model checker [5] is being developed at the University of Utah [7]. Other distributed model checkers have also been implemented [1, 2, 6], however these have limited, or do not use partial order reduction.

The distributed version of PV uses a variant on the original Twophase partial order reduction algorithm [4] and a simple heuristic that may reduce the number of states saved in a distributed verification. The graph search has been changed to make the distributed version of PV perform a breadth first based search.

The distributed Twophase algorithm uses an alternation between a reduction step and a classical breadth first search step. The reduction step executes every commuting [3,4] enabled transition of each process in the model as in the sequential version. The resultant search frontier consists of non-commuting states.

Distributed breadth first search requires sending and receiving of states. The owner of a state is determined when a new state has been returned by Phase-1. States received from other processes are enqueued as though they were generated by the classical breadth first search. States are passed as messages along with information to reconstruct an error trail. A process is assigned a section of the search space using a uniform hash function. Communication only takes place after Phase- 1 .

The distributed version of PV uses a variant on the "Twophase" algorithm that may reduce the amount of unnecessary communication in a distributed verification.

\section{References}

1. Jiri Barnat, Lubos Brim, and Jitka Stribrna. Distributed ltl model-checking in spin. In Proceedings of the 7th International SPIN Workshop, pages 200-216, 2001. LNCS 2057.

2. Hubert Garavel, Radu Mateescu, and Irina Smarandache. Parallel state space construction for model-checking. In Proceedings of the 7th International SPIN Workshop, pages 217-234, 2001. LNCS 2057.

3. Richard J. Lipton. Reduction: A method of proving properties of parallel programs. Сommunications of the ACM, 18(12), December 1975.

4. Ratan Nalumasu and Ganesh Gopalakrishnan. An efficent partial order reduction algorithm with an alternative proviso implementation. Formal Methods in System Design, 20(3):231247, May 2002.

5. Robert Palmer and Ganesh Gopalakrishnan. Partial order reduction assisted parallel modelchecking (full version). Technical report, University of Utah, August 2002.

6. Ulrich Stern and David Dill. Parallelizing the Mur $\phi$ verifier. Formal Methods in System Design, 18(2):117-129, 2001. (Journal version of their CAV 1997 paper).

7. The Utah Verifier group website. http://www.cs.utah.edu/formal_verification.

\footnotetext{
* Supported by NSF Grants CCR-9987516 and CCR-0081406, and a gift from the Intel Corporation.

D.A. Peled and M.Y. Vardi (Eds.): FORTE 2002, LNCS 2529, p. 370, 2002.

(C) Springer-Verlag Berlin Heidelberg 2002
} 